\title{
Estudio de la biodisponibilidad relativa de una formulación multifuente de sulfametoxazol respecto al medicamento referente
}

Ángel Alvarado Yarasca', Alberto Salazar Granara², Neuman Pineda Pérez ${ }^{3}$, Hugo Villanueva Vilchez ${ }^{3}$, Elena Cáceres Andonaire ${ }^{4}$

RESUMEN

Objetivo: Determinar la biodisponibilidad relativa de una formulación multifuente oral de sulfametoxazol de $200 \mathrm{mg} / 5 \mathrm{ml}$ respecto a la formulación referente en Oryctolagus cuniculus $L$ (conejos albinos).

Material y métodos: Las muestras estudiadas consistieron en 25 frascos de suspensión de sulfametoxazol/ trimetoprima multifuente de $200 \mathrm{mg}: 40 \mathrm{mg} / 5 \mathrm{ml}$ asignado con la letra T1 para el lote 11070674; y como referente (R) 25 frascos de suspensión de Bactrim de 200 mg: 40 mg / 5 ml, lote RJ0774.

El protocolo consistió en administrar una dosis oral única de $100 \mathrm{mg} / \mathrm{kg}$ de sulfametoxazol de $200 \mathrm{mg} / 5 \mathrm{ml}$ de cada una de las formulaciones a 12 conejos albinos, después de un ayuno de 12 horas, a través de un diseño abierto, en dos períodos cruzados (T/R), aleatorio y doble ciego, con un intervalo mayor de 5 tiempos de vida media entre cada administración, para determinar posteriormente las concentraciones plasmáticas del fármaco en períodos de tiempo predeterminados hasta las 12 horas por medio de un método espectrofotométrico colorimétrico de diazotación. Con los datos de las concentraciones plasmáticas se construyeron las curvas de biodisponibilidad y de ellas se determinaron el área bajo la curva $\left(A B C_{0-12 h}, A B C_{0 .-\infty}\right), C_{\text {max }} y t_{\text {max }}$.

Resultados: Según análisis estadístico para bioequivalencia, se encontraron: $A B C_{0.12 h} T_{1} / A C_{0.12 h} R I C 90 \% 0,873-1,021, A B C_{0}$.

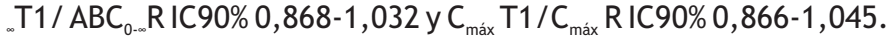

Conclusiones: Los valores encontrados de sulfametoxazol multifuente están dentro del rango aceptable de bioequivalencia propuesto por la OMS y la FDA $(0,80-1,25)$, demostrándose la bioequivalencia del multifuente T1 respecto al referente.

Palabras clave: biodisponibilidad relativa, bioequivalencia, sulfametoxazol, medicamento multifuente, medicamento de referencia.

\section{Study of the relative bioavailability of a multisource formulation of sulfamethoxazole regarding the drug reference}

\section{ABSTRACT}

Objective: To determine the relative bioavailability of an oral formulation of multisource sulfamethoxazole $200 \mathrm{mg} / 5 \mathrm{ml}$ respect to the reference formulation Oryctolagus cuniculus $L$ (albino rabbits).

Material and methods: The samples studied consisted of 25 vials of suspension of sulfamethoxazole / trimethoprim multisource $200 \mathrm{mg}: 40 \mathrm{mg} / 5 \mathrm{ml} \mathrm{T1}$ with the letter assigned to the batch 11070674; and as a reference (R) 25 flasks Bactrim suspension of $200 \mathrm{mg}: 40 \mathrm{mg} / 5 \mathrm{ml}$, RJ0774 batch.

The protocol consisted of a single oral dose of $100 \mathrm{mg} / \mathrm{kg}$ of sulfamethoxazole $200 \mathrm{mg} / 5 \mathrm{ml}$ of each of the formulations to 12 albino rabbits after a 12 hour fasting, through an open design in two cross periods ( $T$ / R), randomized, double-blind study with a greater range of 5 half-lives between each administration, later to determine plasma concentrations of the drug in default until 12 hours by means of a spectrophotometric method periods of time colorimetric diazotization. With the data of plasma, bioavailability curves were constructed, including the area under the curve $\left(A B C_{0.12 h}, A B C_{0 . . .}\right), C_{\max }$ and $t_{\max }$ were determined.

Results: According to statistical analysis for bioequivalence, it was found: $\mathrm{ABC}_{0}{ }^{-}{ }_{12 \mathrm{~h}} \mathrm{~T} 1$ / $\mathrm{ABC}_{0.12 \mathrm{~h}} \mathrm{RCl} 90 \%$ from 0.873 to 1.021 , $\mathrm{AUC}_{0 . \mathrm{m}} \mathrm{T} 1$ / $\mathrm{AUC}_{0 . . .} \mathrm{R} 90 \% \mathrm{Cl} 0.868$ to 1.032 and $\mathrm{C}_{\max } \mathrm{T} 1$ / $\mathrm{C}_{\max } \mathrm{RCl}$ 90\% 0,866-1,045.

Conclusions: The values of multisource sulfamethoxazole found are within the acceptable range of bioequivalence proposed by WHO and the FDA $(0.80-1.25)$, demonstrating the bioequivalence of multisource T1 respect to the reference.

Key words: relative bioavailability, bioequivalence, sulfamethoxazole, multisource drug, drug reference.

1. M.Sc. Unidad de Bioequivalencia y Medicina Personalizada, Centro de Medicina Tradicional y Farmacología. Facultad de Medicina Humana de la Universidad de San Martín de Porres, Lima-Perú.

2. M.D. Dr. Unidad de Bioequivalencia y Medicina Personalizada, Centro de Medicina Tradicional y Farmacología. Facultad de Medicina Humana de la Universidad de San Martín de Porres, Lima-Perú.

3. Q.F, Química Médica. Facultad de Medicina Humana de la Universidad de San Martín de Porres, Lima-Perú.

4. Dra. Facultad de Medicina Humana, Universidad Privada Antenor Orrego, UPAO. 
Ángel Alvarado Yarasca, Alberto Salazar Granara, Neuman Pineda Pérez, Hugo Villanueva Vilchez, Elena Cáceres Andonaire

\section{INTRODUCCIÓN}

En la actualidad para demostrar la equivalencia terapéutica se deben realizar estudios clínicos controlados o estudios de biodisponibilidad, para demostrar la bioequivalencia e intercambiabilidad de los medicamentos multifuentes ${ }^{(1,2)}$.

El término "nombre genérico" es la denominación aceptada por la OMS, bajo las siglas de "Denominación Común Internacional" (D.C.I.) o "International Nonproprietary Names" (I.N.N.) con la cual se nombran los principios activos que llevan los medicamentos similares o copias del original ${ }^{(3)}$. Por lo tanto, un medicamento genérico es aquel que se distribuye o expende rotulado con el nombre $\mathrm{DCl}$ del principio activo, que previamente ha sido desarrollado e inventado por otros, son de bajo costo respecto al innovador, ya que no tiene que demostrar su eficacia y seguridad en ensayos clínicos y tiene la finalidad de aumentar el acceso a la asistencia sanitaria ${ }^{(4,5,6)}$. Mientras que el término de medicamento multifuente $(T)$ indica que son todos los equivalentes farmacéuticos distintos al innovador, tanto similares como genéricos, que pueden o no ser terapéuticamente equivalente, que son elaborados por diferentes fabricantes ${ }^{(1,4,5)}$; y el medicamento innovador, es la especialidad farmacéutica patentada, que ha sido autorizado por primera vez para comercializarse en base a la documentación de calidad, seguridad y eficacia ${ }^{(1)}$.

En tal sentido, el medicamento de referencia $(R)$ es el medicamento innovador, con el cual el medicamento multifuente pretende ser intercambiable, en un estudio de bioequivalencia ${ }^{(1)}$.

La biodisponibilidad (BD) es la medida de la cantidad del ingrediente farmacéutico activo (fármaco) que se absorbe a partir de un medicamento y la velocidad que alcanza la circulación sistémica y se hace disponible en el sitio de acción ${ }^{(1,7,8)}$.

Para demostrar la bioequivalencia entre dos formulaciones distintas de un mismo fármaco, se realiza un estudio clínico de BD relativa o comparativa del medicamento multifuente con el medicamento referente.

En tal sentido, el estudio de bioequivalencia se realiza en voluntarios sanos, en los cuales se cuantifica al ingrediente farmacéutico activo en una matriz biológica como plasma u orina, en los tiempos preestablecidos para obtener los dos parámetros farmacocinéticos primarios, área bajo la curva de concentración plasmática en función del tiempo postadministración $\left(\mathrm{ABC}_{\text {o.t }}\right)$ y la concentración plasmática máxima $^{(2,7)}$. Por lo tanto, la bioequivalencia es la ausencia de una diferencia significativa en la velocidad y cantidad del fármaco que se encuentra disponible en el sitio de acción, cuando se administran dos equivalentes farmacéuticos en la misma dosis molar y en condiciones similares de estudio cinético; son similares en un grado tal que sus efectos son esencialmente el mismo ${ }^{(1,2)}$. Los criterios de aceptación de la bioequivalencia para los parámetros $A B C_{0 . t}, A B C_{0 . \infty}$ y $C_{\text {max }}$ deben basarse en el límite de confianza de $90 \%$ de estas razones, y debe encontrarse en un rango de 0,80 y $1,25^{(1,2,7)}$. En tal sentido un medicamento multifuente intercambiable debe demostrar su bioequivalencia terapéutica con el producto innovador, hasta tal punto de poder ser intercambiado con él, sin merma ni modificación significativa de los efectos terapéuticos y adversos ${ }^{(1)}$.

El sulfametoxazol (2-amino-N-[5-metil-3-isoxazolyl] bencenosulfonamida) es un derivado de las sulfonamidas ${ }^{(9)}$, el cual en forma farmacéutica oral, se debe administrar dos horas antes de los alimentos y con $250 \mathrm{ml}$ de agua, para permitir una rápida velocidad de disolución y una buena absorción a través de la mucosa intestinal ${ }^{(10-15)}$. Esto se produce, ya que son fármacos de clase II, de baja solubilidad y de alta permeabilidad intestinal, según el sistema de clasificación biofarmacéutica ${ }^{(16)}$. Con una dosis estándar, se obtiene una biodisponibilidad del 80 a $90 \%$, con una concentración plasmática máxima de 40 $\mu \mathrm{g} / \mathrm{ml}$, un tiempo máximo de 4 horas $^{(12,13)}$, y una $A B C_{0.30}$ de $516 \mu \mathrm{g} / \mathrm{h} . \mathrm{ml}^{(17)}$. Circula unido a la albúmina plasmática en un $65 \%$ a $70 \%$, siendo su volumen de distribución de $0,20 \mathrm{~L} / \mathrm{kg}$ a 0,36 L/ Kg; y con un tiempo de vida media de 9 a 10 horas $^{(11,14)}$. Se biotransforma a nivel hepático, mediante reacción de fase I, que implica la oxidación del anillo heterocíclico; y por reacciones de fase II de conjugación con $\mathrm{N}$ acetiltransferasa (NAT-1) y NAT-2, originando metabolitos $\mathrm{N}^{4}$-acetil-sulfametoxazol ${ }^{(18)}$. El 25 a $50 \%$ del fármaco inalterado, con sus metabolitos se excretan por filtración glomerular en la orina ${ }^{(11,12)}$.

El objetivo de este estudio fue determinar la biodisponibilidad relativa de una formulación multifuente oral de sulfametoxazol de $200 \mathrm{mg} / 5 \mathrm{ml}$ respecto a la formulación referente en Oryctolagus cuniculus $L$ (conejos albinos). 


\section{MATERIAL Y MÉTODOS}

\section{Material biológico y matriz biológica}

Se emplearon como población de estudio a 12 Oryctolagus cuniculus $L$ (conejos) albinos machos en su variedad Nueva Zelanda, seis en cada secuencia, homogéneos en edad, peso y clínicamente sanos ${ }^{(2)}$. Muestreados de acuerdo a criterios de inclusión y exclusión ${ }^{(2)}$.

La matriz biológica (con el ingrediente farmacéutico activo, IFA) fue extraída y recolectada en los tiempos preestablecidos de los 12 conejos albinos, siendo procesadas en el Laboratorio de Farmacología del Instituto de Investigación de la Facultad de Medicina Humana- USMP, Lima-Perú.

\section{Medicamentos en estudio, adquisición y manejo de} los productos a ensayar

Las muestras estudiadas fueron de origen nacional, las cuales consistieron en:

- Test 25 frascos de suspensión de sulfametoxazol/ trimetoprima multifuente de $200 \mathrm{mg}: 40 \mathrm{mg} / 5 \mathrm{ml}$ de Laboratorio Farmindustria asignado con la letra T1 para el lote 11070674 , RS $N^{\circ} \mathrm{N}-16499$, fecha de vencimiento 10-2017.

- Referencia 25 frascos de suspensión de Bactrim (sulfametoxazol/ trimetoprima) de $200 \mathrm{mg}: 40$ $\mathrm{mg} / 5 \mathrm{ml}$ de Laboratorio Roche, asignado con la letra R para el lote RJ0774, RS N ${ }^{\circ}$ EE-00549, fecha de vencimiento 12-2018.

Se solicitó a un Químico Farmacéutico ajeno al Laboratorio de Farmacología USMP, quien en forma confidencial, confeccionó el ciego, rotulando los frascos como producto A y producto $B$ y anotó su decisión junto al nombre del producto, nombre del laboratorio fabricante, número de lote y fecha de expiración e informó su decisión en sobre sellado a nombre del investigador responsable; sobre que se abrió una vez terminado el estudio; ningún investigador, excepto el profesional mencionado, conoció la identidad de los productos hasta el final del estudio ${ }^{(2,19)}$.

Estos productos se adquirieron en las Boticas ubicadas en los alrededores del Hospital Nacional Arzobispo Loayza del cercado de Lima-Perú. Se usaron reactivos de grado analítico y un estándar primario de sulfametoxazol USP lote SMX06040955, para realizar una curva de calibración.

\section{Métodos}

En la presente investigación, se ha seguido el método modificado de la reacción de diazotación y posterior acoplamiento con el reactivo de Bratton-Marshall, es decir, el N-(1-Naftil) etilendiamina ${ }^{(20,21)}$.

\section{Obtención de la curva estándar de calibración}

Se pesó $10 \mathrm{mg}$ de sulfametoxazol estándar USP y se introdujo en una fiola, aforándola a $100 \mathrm{ml}$ con agua destilada, obteniéndose una solución stock de concentración $0,1 \mathrm{mg} / \mathrm{ml}$.

De la solución stock, se tomó 2 y $5 \mathrm{ml}$ y se incorporaron a las fiolas marcadas como $\mathrm{A}$ y $\mathrm{B}$; a una tercera fiola de $10 \mathrm{ml}$, marcada como $C$, se le adiciona $1 \mathrm{ml}$ de solución stock. A las tres fiolas se les incorporó $8 \mathrm{ml}$ de ácido tricloroacético al 15\%, e inmediatamente se aforó con agua destilada.

Se midió $10 \mathrm{ml}$ de cada una de las soluciones anteriores y se colocó en tubos de vidrios de capacidad nominal de $20 \mathrm{ml}$ marcados como A, B y C; luego se adicionaron los siguientes reactivos en el orden secuencial: $1 \mathrm{ml}$ de solución de nitrito de sodio al $0,1 \%$, después de 3 minutos de reposo se agregó 1 $\mathrm{ml}$ de sulfamato de amonio al $0,5 \%$, se dejó reposar por 2 minutos.

Después de ese tiempo, se agregó $1 \mathrm{ml}$ de solución de $\mathrm{N}$-(1-naftil) etilendiamina. $2 \mathrm{HCl}$ al $0.1 \%$. Inmediatamente, se procedió a filtrar con papel de filtro $595(w-1)$.

A continuación, se realizó las lecturas en el espectrofotómetro UV/Vis 2550 Shimadzu, a una longitud de onda de $545 \mathrm{~nm}$. Para ello, previamente se estandarizó con agua destilada.

Control de calidad biofarmacéutico: prueba de contenido

La prueba de contenido del fármaco se realizó preparando un pool de las suspensiones de sulfametoxazol/trimetoprima.

Luego, se midió un volumen equivalente a $200 \mathrm{mg}$ de sulfametoxazol, el mismo que se introdujo en una fiola, y se aforó a $1000 \mathrm{ml}$ con agua destilada, obteniendo la solución stock.

De la solución stock, se tomó $2,5 \mathrm{ml}$ y se llevó a una fiola de $100 \mathrm{ml}$, inmediatamente, se le incorporó $8 \mathrm{ml}$ 
de ácido tricloroacético al 15\% y se aforó a $100 \mathrm{ml}$ con agua destilada.

Se midió $10 \mathrm{ml}$ de las solución anterior $(0,05 \mathrm{mg}$ de sulfametoxazol) y se colocó en un tubo de vidrio de capacidad nominal de $20 \mathrm{ml}$; luego, se adicionaron los siguientes reactivos en el orden secuencial: $1 \mathrm{ml}$ de solución de nitrito de sodio al $0,1 \%$; después de 3 minutos de reposo, se agregó $1 \mathrm{ml}$ de sulfamato de amonio al $0,5 \%$, dejando reposar por 2 minutos, luego se agregó $1 \mathrm{ml}$ de solución de $\mathrm{N}$-(1-naftil) etilendiamina. $2 \mathrm{HCl}$ al $0,1 \%$. Inmediatamente, se filtró con papel de filtro 595 (w-1). A continuación, se procedió a realizar las lecturas en el espectrofotómetro a una longitud de onda de $545 \mathrm{~nm}$. Para ello previamente se estandarizó con agua destilada.

\section{Diseño del estudio de la bioequivalencia}

Los productos farmacéuticos se administraron a través de un diseño experimental aleatorio, cruzado, comparativo y doble ciego ${ }^{(2,7)}$. Para evaluar la biodisponibilidad y establecer la bioequivalencia del sulfametoxazol, los 12 conejos albinos fueron divididos en dos grupos de 06 cada uno y marcados para distribuirlos de acuerdo al diseño de dos períodos:

En el primer período, se administró una secuencia de medicamento multifuente T1 y medicamento de referencia $\mathrm{R}(\mathrm{T} 1-\mathrm{R})$.

En el segundo período, se administró una secuencia de medicamento R y T1 (R-T1), dos tratamientos (T/R), cruzados, al azar, con una dosis única en cada período, con 6 conejos albinos en cada secuencia ${ }^{(1,7)}$.

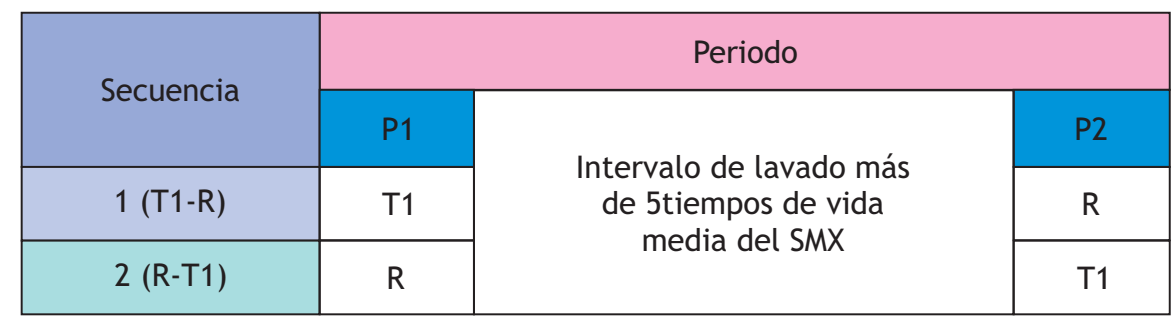

Los dos períodos fueron separados por 15 días de intervalo (transcurridas más de 5 tiempos de vida media del fármaco).

\section{Cuidado de los animales de experimentación}

El día del estudio, se pesó a los conejos y se calculó el volumen de sulfametoxazol a administrar teniendo en cuenta que la dosis es de $100 \mathrm{mg} / \mathrm{Kg}$ de peso de animal de experimentación. Los conejos albinos fueron sometidos a ayuno nocturno de 12 horas, previas a la administración de los tratamientos, permitiéndose la ingesta de agua ad libitum.

En horas de la mañana, seis conejos recibieron una dosis única por vía oral del medicamento T1 y los otros seis, el medicamento R; inmediatamente después se les suministró un volumen estandarizado de $40 \mathrm{ml}$ de agua.

Después de dos horas de la administración de las muestras en estudio, se les suministró agua, y 4 horas después de haberse administrado los medicamentos se les proporcionó los alimentos estandarizados en cantidad y calidad para todos los conejos y en ambos períodos del estudio $^{(1,7)}$.

\section{Recolección de la matriz biológica}

Se extrajo $2 \mathrm{ml}$ de sangre del seno venoso marginal (vena marginal) de la oreja de los conejos a los tiempos preestablecidos de 0,$25 ; 0,5 ; 1,0 ; 2,0 ; 3,0$; 6,$0 ; 8,0$ y 12,0 horas de administrado la suspensión de sulfametoxazol. Las muestras de $2 \mathrm{ml}$ de sangre se recolectaron en tubos de propileno, que contenían $100 \mu \mathrm{L}$ de solución EDTA. El plasma se obtuvo mediante agitación suave del tubo y centrifugado a 2000 rpm por 10 minutos a temperatura ambiente.

\section{Determinación cuantitativa del ingrediente farmacéutico activo en plasma}

El plasma obtenido se adicionó en frascos de vidrio, con $15 \mathrm{ml}$ de agua destilada; luego, se adicionó $4 \mathrm{ml}$ de ácido tricloroacético al $15 \%$ a cada uno de los frascos identificados correctamente.

Se dejó en reposo por espacio de 3 minutos, posteriormente, se filtró; tomando del filtrado $10 \mathrm{ml}$ para introducirlo en tubos de vidrio de $20 \mathrm{ml}$, a los 
cuales se les adicionó, en forma secuencial: $1 \mathrm{ml}$ de solución de nitrito de sodio al $0,1 \%$, después de 3 minutos de reposo, se agregó $1 \mathrm{ml}$ de sulfamato de amonio al $0,5 \%$, dejándolo en reposo por 2 minutos. Después de ese tiempo se agregó $1 \mathrm{ml}$ de solución de $\mathrm{N}$-(1-naftil) etilendiamina. $2 \mathrm{HCl}$ al $0,1 \%$. Inmediatamente se filtró con papel de filtro 595 $(\mathrm{w}-1)$.

El plasma tratado se mantuvo en congelación en una refrigeradora Bosch a $-20^{\circ} \mathrm{C}$ hasta su análisis. Las lecturas se realizaron en el espectrofotómetro, a una longitud de onda de $545 \mathrm{~nm}$. Para ello, previamente se estandarizó con agua destilada.

\section{Análisis farmacocinético}

El análisis farmacocinético se desarrolló después de determinar el orden de la cinética de absorción y eliminación; que, en este caso, se ajusta a una cinética de primer orden, y se asumió un modelo farmacocinético monocompartimental. Con los datos obtenidos, se calcularon los parámetros farmacocinéticos de la biodisponibilidad (concentración plasmática máxima, tiempo máximo, área bajo la curva de concentraciones plasmáticas hasta 12 horas y hasta tiempo infinito; tiempo de vida media y constante de eliminación), para establecer la bioequivalencia del multifuente de acuerdo a la directiva para establecer equivalencia terapéutica de medicamentos ${ }^{(2,7,9)}$.

\section{Análisis de bioequivalencia y estadístico}

Para establecer bioequivalencia se utilizaron valores límites entre 80 a $125 \%$ en relación al producto de referencia, para el intervalo de confianza al $90 \%$ (IC90\%) para la relación de las medias de los parámetros farmacocinéticos $\left(C_{\text {máx }}, A B C_{0-t}\right.$ y $\left.A B C_{0-\infty)}\right)$. Para establecer las posibles diferencias entre los parámetros farmacocinéticos determinados para cada producto farmacéutico en cada animal experimental, se utilizó el test de análisis de varianza multifactorial (ANOVA), estimándose una diferencia estadísticamente significativa para valores de $\mathrm{p} \leq$ 0,05 . Como fuente de variación se consideraron el producto administrado, el período de administración, la secuencia y el efecto residual. Se utilizaron las recomendaciones de las guías de la FDA, tanto para el diseño del estudio como para el análisis farmacocinético y estadístico ${ }^{(1,2,7,22)}$.

\section{RESULTADOS}

El medicamento multifuente ( $\mathrm{T} 1$ ) y referente, ambos en suspensión de $200 \mathrm{mg}$ de sulfametoxazol: $40 \mathrm{mg}$ trimetoprima/5 ml, cumplen con todos los ensayos físicos y químicos, por lo tanto ambos productos son equivalentes farmacéuticos. En la tabla $\mathrm{N}^{\circ} 1$ se registra las concentraciones plasmáticas experimentales promedio en el tiempo, luego de la administración de una sola dosis del medicamento multifuente y del referente.

Tabla 1. Concentraciones plasmáticas promedio en el tiempo postadministración del multifuente T1 y el referente $R$

\begin{tabular}{|c|c|c|}
\hline $\begin{array}{c}T \\
(\mathrm{~h})\end{array}$ & $\begin{array}{c}\mathrm{T}-1 \\
(\mu \mathrm{g} / \mathrm{ml})\end{array}$ & $\begin{array}{c}\mathbf{R} \\
(\mu \mathrm{g} / \mathrm{ml})\end{array}$ \\
\hline 0,0 & 0,00 & 0,00 \\
\hline 0,25 & 0,32 & 0,36 \\
\hline 0,5 & 0,70 & 0,81 \\
\hline 1,0 & 0,96 & 1,03 \\
\hline 2,0 & 0,98 & 1,11 \\
\hline 3,0 & 0,65 & 0,70 \\
\hline 6,0 & 0,32 & 0,28 \\
\hline 8,0 & 0,13 & 0,14 \\
\hline 12,0 & 0,02 & 0,03 \\
\hline
\end{tabular}

La evolución de la concentración plasmática promedio en el tiempo post administración oral de sulfametoxazol T1 y el referente, se exponen en la siguiente figura.

Figura 1. Perfil de la concentración plasmática promedio en el tiempo del multifuente T1 en comparación con el referente $R$

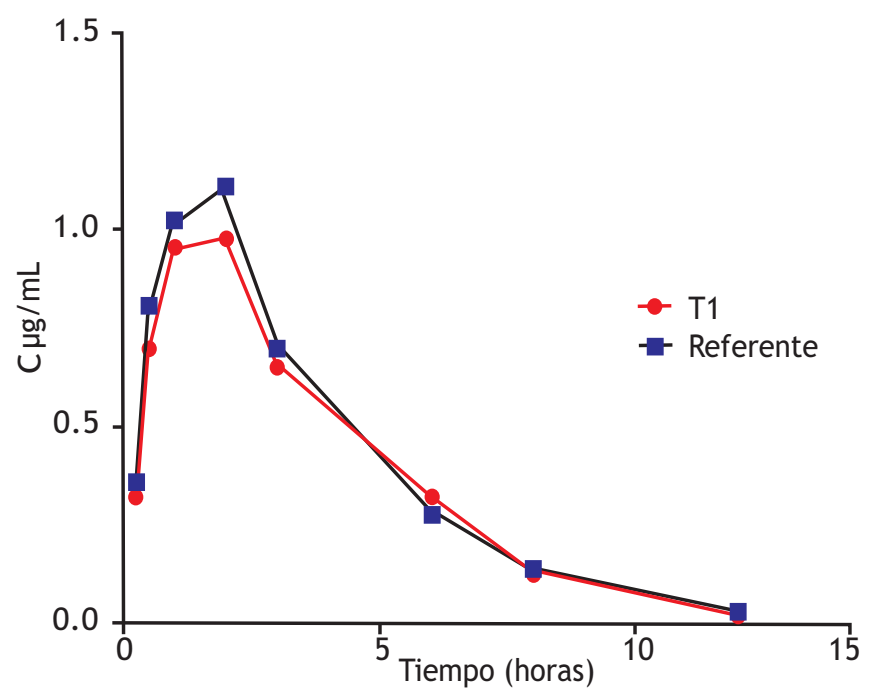


En la siguiente tabla se exponen los parámetros farmacocinéticos primarios de la biodisponibilidad del multifuente $T 1$ y del referente.

Tabla 2. Parámetros farmacocinéticos de sulfametoxazol para la formulación multifuente T1 y referente R

\begin{tabular}{|c|c|c|c|c|c|c|c|c|}
\hline \multirow{2}{*}{$\begin{array}{c}\text { O. } c \\
(n=12)\end{array}$} & \multicolumn{2}{|c|}{$\begin{array}{l}\text { ABC o-12h } \\
(\mu \mathrm{gl} / \mathrm{ml})\end{array}$} & \multicolumn{2}{|c|}{$\begin{array}{c}\mathrm{ABC} 0^{-\infty} \\
(\mu \mathrm{g} / \mathrm{ml} . \mathrm{h})\end{array}$} & \multicolumn{2}{|c|}{$\begin{array}{c}\text { Cmax } \\
(\mu \mathrm{g} / \mathrm{ml})\end{array}$} & \multicolumn{2}{|c|}{$\begin{array}{c}\text { Tmax } \\
\text { (h) }\end{array}$} \\
\hline & $\mathrm{T} 1$ & $\mathbf{R}$ & $\mathrm{T} 1$ & $\mathbf{R}$ & T1 & $R$ & T1 & $R$ \\
\hline Promedio & 4,543 & 4,866 & 4,646 & 4,952 & 1,142 & 1,222 & 1,150 & 1,115 \\
\hline D.E. & 0,425 & 0,563 & 0,510 & 0,577 & 0,096 & 0,181 & 0,272 & 0,225 \\
\hline C.V. \% & 9,37 & 11,57 & 10,98 & 11,66 & 8,47 & 14,85 & 23,67 & 20,20 \\
\hline$M G$ & 4,524 & 4,836 & 4,619 & 4,921 & 1,138 & 1,208 & 1,121 & 1,093 \\
\hline Máximo & 5,030 & 5,630 & 5,340 & 5,700 & 1,360 & 1,420 & 1,670 & 1,430 \\
\hline Mínimo & 3,730 & 4,130 & 3,750 & 4,220 & 1,000 & 0,930 & 0,710 & 0,730 \\
\hline Mediana & 4,670 & 4,710 & 4,765 & 4,900 & 1,145 & 1,300 & 1,065 & 1,100 \\
\hline $1^{\circ} \mathrm{Q}$ & 4,100 & 4,338 & 4,110 & 4,355 & 1,083 & 1,038 & 0,972 & 0,917 \\
\hline $3^{\circ} \mathrm{Q}$ & 4,868 & 5,498 & 5,023 & 5,555 & 1,198 & 1,380 & 1,350 & 1,310 \\
\hline IC95\% mín & 4,273 & 4,508 & 4,322 & 4,585 & 1,080 & 1,106 & 0,977 & 0,971 \\
\hline Ic95\% máx & 4,814 & 5,224 & 4,970 & 5,319 & 1,203 & 1,337 & 1,323 & 1,258 \\
\hline
\end{tabular}

Para establecer la bioequivalencia se utilizaron valores límites entre 80 a 125\% en relación al producto de referencia, para el intervalo de confianza al $90 \%$ (IC90\%) para la relación de las medias de los parámetros

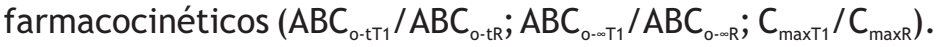

Tabla 3. Análisis de bioequivalencia: multifuente $T 1$ versus referente $R$

\begin{tabular}{|l|r|r|r|}
\hline \multirow{2}{*}{\begin{tabular}{c} 
O. $\begin{array}{c}\mathrm{n}=12 \\
\text { Promedio }\end{array}$ \\
\cline { 2 - 4 }
\end{tabular}} & \multicolumn{3}{|c|}{ Análisis de bioequivalencia } \\
\hline D. E. & 0,947 & 0,950 & $\frac{\text { CmaxT1 }}{\text { Cmax } \mathrm{R}}$ \\
\hline C.V. \% & 0,141 & 0,157 & 0,955 \\
\hline M.G. & 14,97 & 16,56 & 0,172 \\
\hline IC90\% mín & 0,937 & 0,937 & 18,08 \\
\hline IC90\% máx & 0,873 & 0,868 & 0,942 \\
\hline BE respecto al R $\pm 20 \%$ & 1,021 & 1,032 & 0,866 \\
\hline BE según FDA & $0,80-1,20$ & $0,80-1,20$ & 1,045 \\
\hline Relación ABCR/ABCT-1 & $0,8-1,25$ & $0,8-1,25$ & $0,80-1,20$ \\
\hline
\end{tabular}




\section{Discusión}

Los resultados obtenidos en este estudio evidencian que el medicamento multifuente T1 presentó una similar biodisponibilidad relativa $\left(C_{\text {máx }} 1,142 \pm 0,096\right.$ $\mu \mathrm{g} / \mathrm{ml} ; A B C_{0.12}$ de $4,543 \pm 0,425 \mu \mathrm{g} / \mathrm{ml}$.h) en relación al medicamento referente $\mathrm{R}\left(\mathrm{C}_{\text {máx }} 1,222 \pm 0,181 \mu \mathrm{g} / \mathrm{ml}\right.$; $A B C_{0.12}$ de 4,866 $\left.\pm 0,563 \mu \mathrm{g} / \mathrm{ml} . \mathrm{h}\right)$. Lo mencionado anteriormente difiere de lo reportado por Amini, ya que los parámetros farmacocinéticos de la biodisponibilidad relativa de $C_{\text {máx }}$ fue de $41,3 \mu \mathrm{g} / \mathrm{ml}$ y el $A B C_{0 . t}$ fue $516 \mu \mathrm{g} / \mathrm{ml}$.h; es necesario, mencionar que se empleó una metodología distinta a la propuesta por este artículo, ya que lo realizaron por el método de HPLC y en voluntarios sanos ${ }^{(23)}$.

En otro estudio, Chakwenya et al., utilizaron a las alpacas como modelo experimental, reportando que la $C_{\text {máx }}$ fue de $1,9 \mu \mathrm{g} / \mathrm{ml}$ y el $A B C_{o-t}$ fue de 9,1 $\mu \mathrm{g} / \mathrm{ml} . \mathrm{h}^{(24)}$. En este caso, el material biológico y al método utilizado, también difiere de nuestra investigación, ya que nosotros hemos utilizado conejos albinos y el método espectrofotométrico colorimétrico de diazotación.

Para complementar estos estudios, se ha analizado el modelo aditivo tiempo máximo $\left(\mathrm{t}_{\text {máx }}\right)$, observándose que la concentración plasmática máxima del multifuente T1 se alcanza a 1,15 horas, en tanto que el referente lo hace en 1,11 horas; contrastando este parámetro con lo realizado por Hutt et al., quienes reportan que el tiempo máximo es de 1,5 horas para el sulfametoxazol, a pesar de haberla realizado en 12 voluntarios sanos, pero difiere en el valor de la concentración plasmática máxima que era de 1,25 $\mu \mathrm{g} / \mathrm{ml}^{(25)}$.

Para el análisis de varianza multifactorial (ANOVA) se tiene un valor de $p<0,05$, por lo tanto se rechaza la hipótesis nula de bioinequivalencia; mientras que, los intervalos de confianza (IC90\%) para los parámetros $A B C_{0-12}, A B C_{0 . \ldots} y C_{\text {máx }}$ se encuentran dentro del valor medio del medicamento referente $\pm 20 \% 80$ a $120 \%{ }^{(22)} \mathrm{y}$ en el límite de aceptación de 80 a 125\% exigido por la OMS ${ }^{(1)}$ y por la FDA ${ }^{(19)}$, de manera tal que en términos de magnitud del fármaco absorbida a las 12 horas y a tiempo infinito, la formulación multifuente T1 lote 00885297 es bioequivalente e intercambiable con el Bactrim referente lote RJ0468, realizado en Oryctolagus cuniculus $L$ (conejos) albinos machos. Por lo hallado en la presente investigación, amerita un estudio in vivo con voluntarios sanos cumpliendo los criterios de la Organización Mundial de la Salud y la FDA, para corroborar la bioequivalencia del multifuente T1, aplicando el método por cromatografía líquida de alta presión (HPLC).

En conclusión, en base a los resultados obtenidos de los promedios de los parámetros farmacocinéticos de $C_{\text {máx }}, A B C_{0.12 h}$ y $A B C_{0 . .}$ no hay diferencias estadísticamente significativas, y utilizando los criterios de la FDA, los intervalos de confianza al $90 \%$ para la relación de los promedios de los parámetros farmacocinéticos del multifuente $T 1$ y referente $R$, se encuentran dentro de los valores límites del 80 a $125 \%$, por lo que se concluye que el multifuente suspensión de $200 \mathrm{mg}$ de sulfametoxazol:40 mg TMP/ $5 \mathrm{ml}$, es bioequivalente con el producto innovador, a una dosis única administrado por vía oral a conejos albinos machos sanos.

\section{REFERENCIAS BIBLIOGRÁFICAS}

1. World Health Organization. Multisource (generic) Pharmaceutical Products: Guidelines on registration requirements to establish interchangeability. Revision. Working document QAS/14.583. Geneva, 2014.

2. Saavedra I, Ortiz M, Quiñones L, Ortiz M, Sasso J, Varela N, et al. Estudio de biodisponibilidad relativa de una formulación similar de aripiprazol respecto al producto innovador del mercado farmacéutico. Laboratorio de Farmacocinética y Biodisponibilidad. Centro de Investigaciones Farmacológicas y Toxicológicas, Programa de Farmacología, Facultad de Medicina de la Universidad de Chile. 2011.

3. World Health Organization. International Nonproprietary Names for Pharmaceutical Substances (INN). WHO Drug Information, 2011;25( 4)2:413-465.

4. Saavedra I, Saldaña A, Ruminot C. Medicamentos Genéricos. Cuadernos Médico Sociales 2006; 46(3):205-211.

5. Laosa O, Guerra P, López-Durán J, Mosquera B, Frías J. Estudios de bioequivalencia: la necesidad de establecer la fiabilidad de los medicamentos genéricos. Rev Perú Med Exp Salud Pública 2009; 26(4): 553-562.

6. Alfonso R, Andia T, Barbosa T, Watanabe J. Definition and Classification of Generic Drugs Across the World. Appl Health Econ Health Policy 2015; 13 (1):5-11.

7. Saavedra I, Quiñones L, Sasso J, Saavedra M, Gaete L, et al. Estudio de biodisponibilidad relativa entre dos formulaciones orales de micofenolato mofetilo en voluntarios sanos. Rev Med Chile 2011;139:902-908.

8. Chow S. Bioavailability and Bioequivalence in Drug Development. Wiley Interdiscip Rev Comput Stat. 2014 ; 6(4): 304-312.

9. Alonso R, Bernardo R, Valle D, Gonzlilez M, Namur S, Burke V, et al. Bioequivalence of Two Commercial Preparations of Trimethoprim/Sulfamethoxazole: A Randomized, Single-Dose, Single-Blind, Crossover Trial. Clinical Therapeutics 2007;29(2): 326-333.

10. Velasco A, San Román L, Serrano J, Martínez R, Cadavid M. Farmacología Fundamental. Madrid: McGraw-Hill Interamericana; 2002.

11. Mendoza N. Farmacología médica. México: Editorial Médica Panamericana; 2008.

12. Bruton L, Lazo J, Parker K. Goodman-Gilman Las bases farmacológicas de la Terapéutica. 11 ed. Colombia: McGrawHill Interamericana; 2007. 
Ángel Alvarado Yarasca, Alberto Salazar Granara, Neuman Pineda Pérez, Hugo Villanueva Vilchez, Elena Cáceres Andonaire

13. Rang H, Dale M, Ritter J. Farmacología. 4 ed. Madrid: Editorial Harcourt; 2000.

14. Lorenzo P, Moreno A, Leza J, Lizasoain I, Moro M. Velásquez Farmacología Básica y Clínica. Madrid: 17 ed. Editorial Médica Panamericana; 2005.

15. Katzung B. Farmacología básica y clínica. México: 9 ed. Manual Moderno; 2005.

16. Manzo R. Implicancias de la clasificación biofarmacéutica de los fármacos en las regulaciones sobre equivalencia farmacéutica y bioequivalencia y en la práctica farmacéutica. Córdova: Facultad de Ciencias Químicas. Universidad Nacional de Córdova; 2006.

17. Amini H, Ahmadiani A. Rapid and simultaneous determination of sulfamethoxazole and trimethoprim in human by highperformance liquid chromatography. Journal of Pharmaceutical and Biomedical Analysis 2007; 43(3):11461150.

18. Pierce D, Corcoran M, Martin P, Barrett K, Inglis S, Preston P, et al. Effect of $M M X$ mesalamine coadministration on the pharmacokinetics of amoxicillin, ciprofloxacin XR, metronidazole, and sulfamethoxazole: results from four randomized clinical trials. Drug Design, Development and Therapy 2014;8: 529543.

19. Center for Drug Evaluation and Research (CDER). Food and Drug Administration. Guidance for Industry Bioavailability and Bioequivalence Studies for Orally Administered Drug Products - General Considerations Rockville, 2003.

20. Buitrago A, González S, Calderón L. Validación de un método espectrofotométrico para la determinación de clorhidrato de bromhexina en formas farmacéuticas líquidas, basada en la reacción de Bratton-Marshall. Revista de la Facultad de Farmacia 2005;47(1):10-16.

21. Guzmán F. Estudio analítico de sulfamidas, compuestos asociados y productos de degradación mediante nuevos métodos de separación [tesis]. Castilla (España): Universidad de Castilla-La Mancha; 2001. 344 p.
22. Domínguez-Gil A. Los medicamentos genéricos y el 20\%. Revista Española de Economía en Salud 2009; 6:266-267.

23. Amin A, Shahat M, Edeen R, Meshref M. Comparison of Ionpairing and Reversed Phase Liquid Chromatography in Determination of sulfamethoxazole and trimethoprim. Analytical Letters 2008; 41(10): 1878-1894.

24. Chakwenya J, Lakritz J, Tyler J, Fales W, James-Kracke M, Smith K, et al. Pharmacokinetics and bioavailability of trimethoprim-sulfamethoxazole in alpacas. J. Vet Pharmacol Ther. 2002; 25(5): 321-7.

25. Hutt V, Klongmann I, Pabst G, Salama Z, Nieder M, Jaeger H. Studies of the pharmacokinetics and bioavailability of a new trimethoprim/ sulfamethoxazole preparation in healthy volunteers. 1988; 38(9):1347-50.

\section{Fuentes de financiamiento}

Este artículo ha sido financiado por los autores.

\section{Conflictos de interés}

Los autores declaran no tener ningún conflicto de interés.

\section{Correspondencia:}

Ángel Alvarado Yarasca

Dirección: Av. El Corregidor $N^{\circ}$ 1531, Urbanización Los Sirius

Las Viñas, La Molina, Lima 12, Perú

Teléfono: 3652300 anexo 151

Correo electrónico: eaa.alvarado@hotmail.com, aalvaradoy@usmp.pe

Recibido: 26 de abril de 2016 Aprobado: 20 de mayo de 2016 